\title{
The association between neutrophil-to-albumin ratio and mortality in patients with cardiogenic shock: a cohort study
}

\section{Yangpei Peng}

Wenzhou Medical University Second Affiliated Hospital https://orcid.org/0000-0002-8122-3374

\section{Yangjing Xue}

Wenzhou Medical University Second Affiliated Hospital

Jinsheng Wang

Wenzhou Medical University Second Affiliated Hospital

Huaqiang Xiang

Wenzhou Medical University Second Affiliated Hospital

Kangting Ji

Wenzhou Medical University Second Affiliated Hospital

Jie Wang ( $\mathbf{3 9 3 5 5 1 0 6 2 @ q q . c o m ) ~}$

https://orcid.org/0000-0003-3357-5743

Cong Lin

Wenzhou Medical University Second Affiliated Hospital

\section{Research article}

Keywords: Neutrophil-to-albumin ratio, Cardiogenic shock, Intensive care unit

Posted Date: April 22nd, 2020

DOI: https://doi.org/10.21203/rs.2.16002/v2

License: (c) (1) This work is licensed under a Creative Commons Attribution 4.0 International License.

Read Full License 
The authors have withdrawn this preprint from Research Square 